CAHIERS DE

NARRATOLOGIE

\section{Cahiers de Narratologie}

Analyse et théorie narratives

37 | 2020

Approches transmédiales du récit dans les fictions contemporaines

\title{
Le régime cathartique à l'ère du storytelling
}

Antonino Sorci

\section{(2) OpenEdition}

Journals

Édition électronique

URL : http://journals.openedition.org/narratologie/10633

DOI : 10.4000/narratologie.10633

ISSN : 1765-307X

Éditeur

LIRCES

Référence électronique

Antonino Sorci, « Le régime cathartique à l'ère du storytelling », Cahiers de Narratologie [En ligne], 37 |

2020, mis en ligne le 04 septembre 2020, consulté le 06 septembre 2020. URL : http://

journals.openedition.org/narratologie/10633; DOI : https://doi.org/10.4000/narratologie.10633

Ce document a été généré automatiquement le 6 septembre 2020 .

Article L.111-1 du Code de la propriété intellectuelle. 


\title{
Le régime cathartique à l'ère du storytelling
}

\author{
Antonino Sorci
}

Depuis la publication de l'ouvrage de Christian Salmon Storytelling, la machine à fabriquer des histoires et à formater les esprits ${ }^{1}$, les spécialistes en sciences humaines et sociales ont désormais pris conscience du recours constant, de la part des politiciens, à des stratégies et à des techniques narratives. Il s'agira ici moins d'analyser les techniques narratives employées dans le discours politique des partis dits «populistes» que de souligner un phénomène qui n'a pas été, à notre connaissance, suffisamment exploré, à savoir que les politiciens "populistes» semblent s'être inspirés du modèle de récit énoncé par Aristote dans sa Poétique pour forger leurs narrations. Une exploration plus approfondie de ce phénomène peut suggérer deux ensembles de considérations. D'une part, la coïncidence entre la manière de forger les histoires décrites par Aristote et celle adoptée par ces politiciens confirme leur prestige auctorial. Si ce lien existait réellement, leur capacité à attirer un grand nombre d'électeurs dépendrait aussi de la maitrise que ces politiciens ont d'un nombre de principes et de règles de composition qui, depuis Aristote, permettent de juger qu'une œuvre est particulièrement réussie. D'autre part, une analyse de ce phénomène pourrait s'avérer particulièrement précieuse dans la tâche de pointer les implications idéologiques que comporte l'assimilation des principes du modèle aristotélicien de la narrativité tant pour l'auteur des narrations que pour les auditeurs auxquelles celles-ci s'adressent. Cela pourrait se révéler utile surtout pour examiner sous un angle différent certaines considérations qu'on continue encore de nos jours à tenir pour assurées, dont par exemple le fait que les narrations favorisent naturellement la cohésion et l'intégration sociale et que leur utilisation s'avère essentielle dans la tâche commune de vouloir « réparer le monde $~^{2}$. Bien entendu, il ne s'agira pas ici de remettre en cause la valeur pédagogique et cognitive d'un grand nombre de narrations, mais seulement de considérer l'hypothèse selon laquelle celles-ci, en plus de pouvoir être utilisées comme des outils pour stimuler le dialogue social, peuvent aussi, dans certains cas, se révéler des instruments efficaces dans le but de consolider les idées reçues et de renforcer une culture du conformisme. En particulier, l'axe qui, depuis la perspective aristotélicienne, constitue l'armature de 
tout récit, à savoir le rapport étroit qui relie le muthos à la catharsis des émotions, peut être évalué d'une façon différente si on la rapproche de la façon dont les politiciens «populistes » forgent leurs histoires. À ce propos, nous observerons que le lien qui unit le «tout » organisé par l'« agencement des faits en système » à l'« épuration » que la narration est capable de réaliser chez les auditeurs peut être exploité dans certains cas pour propager des versions faussées de certains états de fait, lesquelles s'inspirent notamment d'une série de "théories du complot» particulièrement convaincantes. Afin de mettre en évidence tous ces aspects, nous nous proposons ici d'analyser quelques cas tirés de l'actualité politique en France et en Italie afin d'étudier la capacité des politiciens "populistes » à forger des narrations dont l'« arrangement des faits » serait censé toucher la sphère émotionnelle des auditeurs au point de provoquer un véritable soulagement chez ces derniers.

2 L'analyse de la vocation aristotélicienne des politiciens dits "populistes" ne représentera pas le seul thème que nous aborderons dans cette étude. Nous souhaitons également questionner les implications idéologiques qu'engendre l'adoption de la perspective aristotélicienne de la narrativité. Il s'agira notamment de souligner l'existence d'un véritable "régime cathartique " qui gouverne notre façon de forger nos histoires. Nous défendrons la thèse selon laquelle le retour des « grands récits » que nous connaissons en époque actuelle posséderait un lien avec la disparition de la culture de gauche qu'Enzo Traverso observe dans son ouvrage Mélancolie de gauche $e^{3}$ : la diffusion massive d'un nombre conséquent de "grands récits » forgés selon les règles de composition énoncées par Aristote dans la Poétique aurait permis l'instauration d'un " régime cathartique » qui ne laisserait pas de place à l'élaboration et à l'interprétation de formes de narration alternatives de caractère ouvertement anti-cathartique et antiaristotélicien.

\section{Considérations générales au sujet des notions de muthos et de catharsis}

Avant d'aborder l'analyse des extraits des discours des politiciens dits "populistes ", nous souhaitons exposer quelques thèses récentes au sujet des notions de muthos et de catharsis. Depuis la publication de Temps et Récit, c'est une pratique commune de traduire le muthos aristotélicien par « mise en intrigue ». Chez Ricœur, cette traduction ouvre à la compréhension de la pratique narrative entendue comme une des modalités principales à travers lesquelles l'être humain serait capable de donner une configuration aux événements vécus. L'expérience humaine possède une dimension purement narrative que le philosophe n'hésite pas à définir comme une « demande de récit » :

À cette objection, j'opposerai une série de situations qui, à mon avis, nous contraignent à accorder déjà à l'expérience en tant que telle une narrativité inchoative qui ne procède pas de la projection, comme on dit, de la littérature sur la vie, mais qui constitue une authentique demande de récit. Pour caractériser ces situations je n'hésiterai pas à parler d'une structure pré-narrative de l'expérience ${ }^{4}$.

Depuis cette perspective, la narrativité jouerait un rôle majeur dans la configuration des faits et des événements qui caractérisent l'expérience de vie quotidienne des individus. Selon Ricœur, le modèle aristotélicien de la Poétique, centré sur la prédominance du binôme mimesis-muthos, s'affirme comme un modèle de concordance 
discordante qui indique la façon dont les individus utilisent la pratique narrative afin d'appréhender les événements vécus. Ce modèle est tout d'abord un modèle de concordance, du fait que la narration « est caractérisée par trois traits : complétude, totalité, étendue appropriée ${ }^{5} »$. Mais ce modèle inclut également une discordance entre les éléments de l'histoire racontée, puisque la «mise en intrigue» définit une succession épisodique capable d'engendrer une «incertitude temporaire" chez l'interprète. C'est d'ailleurs en ces termes que Raphaël Baroni définit la "mise en intrigue », qui se trouve au centre de sa conception de la narrativité. La configuration du muthos aristotélicien permet l'élaboration d'une tension narrative qui rythme l'interprétation des événements appréhendés. L'intrigue s'articule selon trois phases principales: la phase du noud "produit un questionnement qui agit comme un déclencheur de la tension $»^{6}$, l'interprète est amené à ce stade à se poser des questions à propos de la suite de l'histoire. Une seconde phase, celle du retard, se caractérise par la dialectique entre incertitude et anticipation du dénouement attendu. L'intrigue se termine au moment du dénouement, à savoir le moment dans lequel l'interprète établit une configuration précise des événements appréhendés. C'est à ce moment que ce dernier est en mesure de donner une réponse au questionnement suscité par le nœud. La répartition des événements selon la charnière constituée par le couple nœud/ dénouement est rendue possible grâce au rapport d'équivalence qui s'établit entre la représentation des actions, des personnages et l'agencement des faits. Les actions des personnages, comme le souligne Propp dans sa Morphologie du conte, constituent de véritables «fonctions" au sein de l'intrigue. Puisque «l'action ne peut être définie en dehors de sa situation dans le cours du récit $»^{7}$, l'interprète, afin de saisir la signification des actions des personnages de l'histoire, doit être capable de reconstituer les liens logiques et causaux qui relient celles-ci à l'intérieur de l'intrigue. L'interprétation d'un récit, selon l'optique aristotélicienne, constitue donc une opération qui implique un effort cognitif de la part de l'interprète. Ce dernier est amené à ressentir une tension provisoire produite par l'incertitude à l'égard du destin des protagonistes de l'histoire, qui disparaît une fois reconnu l'ordre des actions et la configuration du récit.

5 La traduction du terme muthos par "mise en intrigue» possède en outre des implications d'ordre terminologique importantes. Comme le souligne notamment Johanne Villeneuve, le terme "intrigue" est particulièrement riche d'un point de vue sémantique. Outre désigner la façon dont les événements de l'histoire sont agencés, il peut également être interprété comme synonyme de « complot » :

Le substantif intrigue, en usage encore aujourd'hui pour désigner l'ensemble des événements formant le nœud d'une pièce de théâtre ou d'un film, est également appelé à signifier un ensemble de combinaisons secrètes et compliquées visant à faire réussir ou avorter une affaire. Cette dernière signification rejoint le domaine de la conspiration et du complot. En anglais, c'est un terme encore plein de cette résonance du complot qui s'est imposé en poétique, soit plot, tandis que to intrigue évoque l'usage d'une influence secrète, l'action de comploter et d'intriguer; plot renvoie aussi à la fourberie, à l'illicite ${ }^{8}$.

6 Intriguer quelqu'un au moyen d'une histoire ne représenterait pas, d'un point de vue axiologique, une opération neutre : la «mise en intrigue » doit être entendue comme une action comprenant des stratégies auctoriales qui peuvent engendrer des effets considérables sur les interprètes. Ces effets ne se limitent pas à toucher la sphère cognitive de l'interprète, mais également les sphères psychologique et émotionnelle. $C^{\prime}$ est la raison pour laquelle la notion de muthos ne peut être comprise pleinement que 
si on la met en rapport avec la notion de catharsis. Nous souhaitons nous attarder notamment sur l'analyse de quelques interprétations de ce terme dont, ne l'oublions pas, Aristote ne fournit pas de définition précise. Dans l'édition de la Poétique dirigée par Jean Lallot et Roselyne Dupont-Roc, la catharsis est associée au plaisir mimétique, fondé sur la « reconnaissance des formes » et lié à l'art de " composer des intrigues » :

Si donc la tragédie peut « épurer » les émotions qu'elle éveille chez le spectateur et ainsi lui donner du plaisir et non de la peine, c'est en tant qu'elle offre à son regard des objets eux-mêmes épurés [...]. La katharsis tragique est le résultat d'un processus analogue: mis en présence d'une histoire (muthos) où il reconnaît les formes, savamment élaborées par le poète, qui définissent l'essence du pitoyable et de l'effrayant, le spectateur éprouve lui-même la pitié et la frayeur, mais sous une forme quintessenciée, et l'émotion épurée qui le saisit alors et que nous qualifierons d'esthétique s'accompagne de plaisir'.

Par sa capacité à provoquer la transformation des émotions négatives évoquées par la narration en une source de plaisir, la catharsis permet de renforcer les convictions de l'interprète à propos d'un nombre de formes « savamment élaborées par le poète » qu'il reconnaît comme familières. C'est ainsi que la catharsis est généralement considérée par la plupart des spécialistes comme un mécanisme d'adhésion affective puissant, qui se révèle particulièrement utile dans la tâche de faciliter la diffusion d'un nombre de messages caractérisés par leur haute valeur anthropologique et sociale. En particulier, Hans Robert Jauss est revenu plusieurs fois sur le sujet, en soulignant l'importance du rôle joué par la catharsis dans la transmission d'un nombre de normes de comportement social :

Catharsis désigne un troisième aspect de l'expérience esthétique fondamentale: dans et par la perception de l'œuvre d'art, l'homme peut être dégagé des liens qui l'enchaînent aux intérêts de la vie pratique et disposé par l'identification esthétique à assumer des normes de comportement social [...]. La jouissance cathartique joue alors - pour citer Freud - le rôle d'appât et peut induire le lecteur ou le spectateur à assumer beaucoup plus facilement des normes de comportement et à se solidariser davantage avec un héros, dans ses exploits comme dans ses souffrances ${ }^{10}$.

Pour résumer, muthos et catharsis sont deux notions fondamentales du modèle aristotélicien de la narrativité. Elles sont des notions étroitement interconnectées, tant sur un plan idéologique que sur un plan fonctionnel. Le rapport d'échange qui s'instaure entre ces deux composantes de la narrativité est fondé sur le rôle joué par le dispositif de la reconnaissance qui s'instaure au cours de l'interprétation d'un texte narratif.

\section{Les intrigues des politiciens «populistes »}

9 À la lumière de ce qui précède, nous souhaitons analyser quelques extraits tirés de l'actualité politique en France et en Italie et qui concernent notamment le discours politique des partis dits "populistes». On sait que les politiciens "populistes» ont constamment recours à des stratégies narratives particulières dans le but de convaincre un plus grand nombre d'électeurs potentiels. Ce qui n'a pas été, à notre connaissance, suffisamment exploré c'est la proximité entre la vision aristotélicienne $\mathrm{du}$ récit et la façon dont ces politiciens forgent leurs histoires. Grâce au recours constant à des moyens rhétoriques efficaces, les politiciens "populistes " arrivent à obtenir une implication émotionnelle pleine de la part de leurs auditeurs. La stratégie narrative est claire : ces politiciens forgent de véritables intrigues structurées à l'image 
des muthoi aristotéliciens. On constate, tout d'abord, qu'un élément d'incertitude est introduit dans le discours au moment d'évoquer la prétendue « décadence " qu'est en train de vivre le "peuple » auquel ceux-ci se référent. Voici un extrait d'un discours que Marine Le Pen a prononcé à Bordeaux en janvier 2012 qui est emblématique de cette démarche :

Voilà ce qui nous invite à cette terrible pensée : l'idée que la fin de la France serait désormais plus probable que son redressement. Rien ne change et chaque jour un peu plus le pays s'enfonce dans le sentiment d'un déclin inexorable, d'une perte d'influence, d'une rétrogradation, d'une descente aux enfers présentée comme inéluctable ${ }^{11}$.

Le scénario présenté ici vise à instiller une forte inquiétude chez les auditeurs: l'évocation d'un possible " déclin » et d'une probable « descente aux enfers » du peuple français a comme objectif de causer la déstabilisation initiale des auditeurs. Les politiciens "populistes » font appel à des mécanismes propres à la pratique narrative comme l'empathie ressentie envers les membres des communautés exposés à de multiples attaques. Saisi par l'effroi, le destinataire du message cherche spontanément des appuis auxquels s'accrocher afin d'éviter de s'enfoncer davantage en un sentiment de détresse. C'est ainsi que le politicien " populiste » lui vient en aide en lui offrant un soutien stable sur lequel il pourra s'appuyer : tout comme le recours à un imaginaire mythique, les discours des politiciens "populistes» sont souvent centrés sur la présence d'un narrateur aux traits messianiques. L'identification entre le narrateur et l'auteur du discours est ici totale: le narrateur s'affirme comme un nouveau messie capable de sortir le «peuple» de son impasse. Le narrateur représente le « héros » qui luttera jusqu'au bout de ses forces afin de protéger les citoyens mis en péril par les attaques des "ennemis du peuple ». La présence du héros constitue un contrepoids à celle de l'ennemi, à l'intérieur d'une stratégie de «diabolisation» de l'adversaire politique. C'est ainsi que celui-ci est transformé par les politiciens «populistes » en démon avec lequel il serait impossible de se confronter. Comme l'a souligné PierreAndré Taguieff à propos du discours des nationalistes identitaires : « on ne discute pas avec des démons, avec leurs représentants ou leurs complices, on ne dialogue pas avec eux ; on les combat, et ce afin de les supprimer ${ }^{12} »$. La narration de ces politiciens, par conséquent, est souvent investie d'une portée moralisatrice très puissante. L'histoire racontée porte sur une bataille qui voit les forces du Bien s'opposer constamment à celles d'un Mal menaçant la vie des citoyens. Les solutions trouvées dans cette optique, au lieu de constituer des prises de position idéologiques particulières, s'affirment comme des mesures de «bon sens». Voici un exemple, qui date de juin 2016, de comment le chef du Mouvement 5 Étoiles Luigi di Maio décrit l'action politique de ses collègues élus à la chambre des députés du parlement italien :

Les conseillers élus du Mouvement 5 Étoiles approuveront à la Chambre des députés toute proposition de bon sens, quel que soit le candidat qui la présente : les bonnes idées ne sont ni de droite ni de gauche ${ }^{13}$.

11 Mais l'incertitude instaurée par l'intrigue, à l'instar du modèle aristotélicien de la Poétique, s'avère n'être que provisoire : l'inquiétude instillée chez l'auditeur est destinée à disparaitre au moment du dénouement attendu et à se transformer en plaisir intense. Plus grande sera la virulence à travers laquelle l'histoire est racontée plus puissante sera le plaisir que l'auditeur prendra à reconnaitre la forme globale de l'intrigue. Voici comment une députée du parlement italien, Lucia Borgonzoni, invoque le retour au pouvoir de son parti, la Ligue, parti de l'extrême-droite italienne, après l'avoir cédé à 
une coalition formée par le Mouvement 5 Étoiles et le parti de centre-gauche italienne, le Parti Démocrate, intervention qui date de septembre 2019 :

Bientôt, il y aura des élections régionales [...] et vous devrez vous confronter avec le vote des Italiens qui vous ont rejetés lors de toutes les élections de ces dernières années [...] On ne peut pas fuir éternellement. Et lorsque vous rendrez la parole aux citoyens, vous serez anéantis pour toujours ${ }^{14}$.

La fin de l'extrait coïncide avec le dénouement attendu : au moment d'apprendre que les « ennemis du peuple » seront « anéantis pour toujours » les auditeurs ressentent un plaisir intense provoqué par la catharsis des émotions éprouvées au cours de l'acte interprétatif. Bien entendu, afin de ressentir pleinement la catharsis espérée, le destinateur du message doit partager les mêmes convictions de l'auteur de ces intrigues. C'est la raison pour laquelle, contrairement à l'opinion répandue en sciences humaines et sociales qui conçoit la catharsis engendrée par les narrations comme une des composantes fondamentales du vivre-ensemble, nous tenons à souligner que celleci peut être aussi l'expression d'une volonté ferme de la part de l'auteur d'amener les interprètes à une acceptation acritique des narrations proposées. En effet, notre hypothèse consiste à interpréter la catharsis comme une forme de réaction émotive produite « artificiellement " par la dimension «chrono-logique» du muthos. Le plaisir qu'elle suscite, l'épuration des sensations, dépend de la volonté de la part de l'auteur et de l'interprète de se complaire artificiellement dans un système de normes dont ils reconnaissent l'exemplarité. La logique du muthos servirait à l'auteur pour véhiculer une vision du monde fondée sur le respect des normes sociales et sur la légitimation d'un code de comportement éthique que l'interprète et l'auteur reconnaissent comme familier. La recherche de la catharsis serait donc fondée sur la volonté, tant de la part l'auteur que de l'interprète, de partager une vision conformiste de la réalité sociale dans laquelle ils sont situés. Peu importe que cette vision adhère ou non à un état de fait réel, ce qui importe c'est que la narration puisse contribuer à renforcer le lien qui unit les politiciens "populistes " au "peuple " auquel ils se réfèrent. C'est la raison pour laquelle l'entente qui se crée entre l'auteur et l'interprète au cours de l'acte narratif peut être considérée comme un facteur décisif dans la diffusion massive d'un nombre de fake news et de narrations contrefactuelles de la réalité. Si les «théories du complot » connaissent de nos jours un si grand succès, ceci peut s'expliquer aussi par le pouvoir cathartique possédé par ce genre de narrations. Ces théories s'avèrent d'une grande utilité dans la tâche tant de rassembler le "peuple» contre les groupes d'individus qui sont accusés de conspirer à son insu que de fédérer celui-ci autour de la figure d'un leader incarnant le vrai «esprit du peuple». C'est la raison pour laquelle plusieurs auteurs ont souligné la présence dans le discours de certains politiciens " populistes ", dont Marine Le Pen, de plusieurs allusions à des "théories du complot " particulièrement convaincantes. Cécile Alduy et Stéphane Wahnich ont souligné dans leur ouvrage Marine Le Pen prise aux mots la fonction légitimante de la figure de l'auteur possédée par les « théories du complot » :

À cette fonction explicative ou cognitive qui récompense l'auditoire s'ajoute une fonction légitimante qui valorise l'énonciatrice. Chez Marine Le Pen, la dimension occulte de complots tramés dans l'ombre par des sectes elles-mêmes secrètes a presque disparu, mais le secret revient sous la forme plus rationnelle du mensonge : «C'est un mensonge d'État qui traduit l'adhésion de nos gouvernants à un véritable projet clandestin donc antidémocratique d'installation massive d'une immigration de peuplement $»^{15}$. 
Quoi qu'il en soit, les théories du complot et les narrations cathartiques sont utilisées par ces partis dans un seul but : comme le souligne Jan-Werner Müller, l'objectif des politiciens "populistes» est celui de s'assurer le «monopole de la représentation ». Puisque les partis populistes visent dans leurs discours à synthétiser la vision du monde des "vrais citoyens", ceux-ci ne peuvent pas être considérés à proprement parler des «partis comme les autres»:

Si les partis populistes ne sont donc en rien des partis comme les autres, c'est en raison de leur revendication morale d'un monopole de la représentation [...] cette idée selon laquelle il serait possible de représenter la totalité (et qui entre en opposition avec la fragmentation politique d'une société) entre en contradiction avec la définition même de ce qui est un parti, qui est toujours particulier, partiel et partial - comme nous le dit déjà le nom même de parti : un parti n'est qu'une «partie » et ne représente pas le « tout $»^{16}$.

Devant l'ambition des partis "populistes» de vouloir s'assurer le monopole de la représentation à l'aide de narrations cathartiques, il nous semble légitime de se demander s'il y a des raisons d'ordre idéologique et politique derrière le retour des "grands récits » et la disparition des récits non-aristotéliciens que nous constatons de nos jours: sommes-nous entrés dans un «régime cathartique » qui gouverne notre façon de forger nos histoires?

\section{Le régime cathartique à l'ère du storytelling}

Nous avons observé dans la partie qui précède la proximité qui existe entre la manière dont les politiciens "populistes » forgent leurs histoires et les principes énoncés par Aristote dans sa Poétique. Ces politiciens révèlent leur assimilation des règles de composition qui, depuis Aristote, permettent de juger une narration comme particulièrement réussie. Si le discours "populiste» semble avoir intégré un nombre d'éléments appartenant au domaine narratif nous pourrions nous demander si, en retour, la façon dont les récits sont élaborés et diffusés de nos jours reflète la manière «populiste » de préparer un discours. Ce qui saute aux yeux c'est que les auteurs des narrations contemporaines qui remportent un grand succès auprès du public s'inspirent directement du modèle aristotélicien de la narrativité pour forger leurs histoires. Des séries-télés aux bandes dessinées, en passant par les blockbusters que les grosses productions de l'industrie cinématographique adressent à un large auditoire, il est devenu de plus en plus rare que des narrations qui n'ont pas été conçues en suivant les règles de composition énoncées par Aristote soient susceptibles de rencontrer un grand succès populaire. Ce recours constant à des narrations fondées sur la dimension chrono-logique du muthos répond à un besoin de catharsis de plus en plus croissant. Ce dernier se fonde principalement sur le rapport de confiance et de loyauté qui s'instaure entre les interprètes et les auteurs des narrations. En effet, au même titre que les politiciens " populistes », les auteurs des séries-télés fondent leurs stratégies narratives sur le programme de "fidélisation" des sujets auxquels ceux-ci s'adressent. Ces auteurs élaborent de "grands récits " qui sont ensuite segmentés et proposés aux spectateurs selon une programmation qui doit seconder l'envie de ces derniers de connaître "la suite de l'histoire ». Cet aspect a récemment été souligné par Anaïs Goudmand: "Les processus d'actualisation se caractérisent par le morcellement: contrairement à la lecture d'un roman ou au visionnage d'un film qui constituent des récits complets, le récepteur est soumis à des stratégies visant à le "fidéliser" en 
programmant la sortie de chaque nouvel épisode à des intervalles réguliers, et l'actualisation du récit se fait sur du long terme $»^{17}$. Entre les auteurs de ces épisodes et les spectateurs se crée donc une entente qui rappelle celle qui s'établit entre les politiciens «populistes » et leur auditoire : dans les deux cas le processus interprétatif se fonde sur la volonté de partager une vision du monde commune et de resserrer le lien qui unit l'auteur à l'interprète. La différence entre ces deux genres d'interaction narrative concerne la figure de l'auteur: une personne physique aux traits reconnaissables dans le cas du discours "populiste» et la maison de production identifiable grâce au logo auquel celle-ci est associée dans le cas des séries-télés. Ce qui ne change pas c'est le dispositif cathartique qui sous-tend le rapport qui se crée entre auteur et interprète : à titre d'exemple, les spectateurs de la maison de production Netflix s'attendent un genre de narrations qui soient forgées en respectant les particularités du «style Netflix » et, de retour, les auteurs des séries-télés Netflix se proposent d'offrir des contenus qui puissent seconder les attentes des spectateurs ${ }^{18}$. L'empathie qui se crée entre la maison de production et les spectateurs est comparable à celle qui se produit entre les grandes marques et les consommateurs. Naomi Klein dans son fameux ouvrage No Logo souligne cet aspect en mentionnant le cas de la marque Starbucks :

Scott Bedbury, vice-président marketing de Starbucks, reconnut ouvertement que " les consommateurs ne voient pas de différence énorme entre les produits ", et que pour cette raison, les marques doivent «établir des liens émotionnels » avec leurs clients au moyen de "l'Expérience Starbucks». Les gens qui font la queue chez Starbucks, écrit le P.D.G. Howard Shultz, ne viennent pas que pour le café. « C'est le côté romantique de l'expérience du café, le sentiment de chaleur et de communauté que trouvent les gens dans les cafés Starbucks ${ }^{19}$.

Tels les clients de Starbucks, les spectateurs des séries-télés Netflix souhaitent avant tout appartenir à la communauté formée par tous les spectateurs/consommateurs des produits Netflix. La possession d'une charge cathartique puissante représente donc un point commun important entre les narrations «populistes » et un grand nombre de récits contemporains. Mais ce qui rapproche le plus les politiciens "populistes » des auteurs des séries-télés, c'est leur volonté commune de s'assurer le «monopole de la représentation ». Les maisons de production des séries-télés ont l'ambition de monopoliser tout le marché de l'audiovisuel en englobant ainsi une bonne partie de l'imaginaire contemporain. Ce processus a comme conséquence directe le fait de disqualifier toute narration qui ne respecte pas les normes de qualité du «style Netflix ». Selon cette perspective, toute narration qui ne serait pas produite par ces maisons de production n'arriverait pas à garantir ces standards de qualité en perdant ainsi tout son caractère narratif : toute narration qui ne serait pas produite par ces maisons de production se transformerait automatiquement en un non-récit. C'est la raison pour laquelle nous souhaitons porter un regard plus sévère sur la prétendue valeur éducative et morale des séries-télés que celui porté par de nombreux chercheurs en sciences humaines et sociales dont notamment Sandra Laugier. Pour notre part, le fait que " les séries changent notre quotidien », qu'elles soient " constitutives de formes de vie humaine » et qu'« elles donnent accès à la réalité de façon nouvelle, mais aussi à une formation morale $»^{20}$ représente davantage un danger pour la vie commune qu'une opportunité. Contre les tendances hégémoniques des auteurs des récits cathartiques, nous préférons plutôt souligner la valeur éthique et épistémologique possédée par les narrations non-aristotéliciennes dans la tâche d'empêcher la diffusion des idées reçues et de lutter contre le conformisme. Les récits qui semblent appartenir à une époque 
lointaine, celle qui est caractérisée par le défi aux formes conventionnelles d'écriture narrative, par la volonté de limiter l'autorité de l'auteur à l'intérieur du processus interprétatif, d'établir une communication d'expériences fondée sur la distance critique qui doit séparer l'auteur de l'interprète, en deux mots les récits appartenant à l'époque des narrations non-cathartiques du Nouveau Roman, des narrations post-modernes et de l'écriture testimoniale, possèdent à nos yeux une charge subversive supérieure à celle contenue dans les "grands récits » contemporains. Ces narrations « sans catharsis ", comme le souligne Julia Kristeva au sujet de l'œuvre de Marguerite Duras, nous apprennent à mieux reconnaître le sens d'insatisfaction auquel nous sommes quotidiennement confrontés et qui se trouve accentuée par l'homologation forcée aux normes dictées par l'économie du marché: «littérature de nos maladies, elle accompagne les détresses certes déclenchées et accentuées par le monde moderne, mais qui s'avèrent essentielles, transhistoriques ${ }^{21} »$. La mise en valeur de ces formes d'écriture apparaît d'autant plus urgente alors que nous sommes confrontés au risque concret que les politiciens "populistes » et les maisons de production audiovisuelle puissent réellement s'assurer définitivement le «monopole de la représentation ». En effet, ce que nous constatons de nos jours c'est que les histoires conçues à l'instar du modèle aristotélicien de la Poétique ont obtenu une emprise telle sur notre vie quotidienne qu'il ne serait pas exagéré de parler à ce propos d'un «régime cathartique » gouvernant l'imaginaire contemporain. Ce régime façonne les goûts et les opinions du public au point de l'induire à s'intéresser uniquement aux récits aristotéliciens et le pousse à négliger la lecture de toute narration qui ne soit pas fondée sur la recherche d'un effet cathartique. Il convient à ce propos d'observer que le maigre succès que les récits non-aristotéliciens obtiennent auprès du public de nos jours coïncide avec la disparition de l'imaginaire de gauche qu'Enzo Traverso signale dans son ouvrage récent Mélancolie de gauche. La force d'une tradition cachée (XIX - XXI siècle). La dimension mélancolique qui, selon Traverso, représente le trait distinctif de la «culture de gauche", se trouve être occultée de nos jours par la rhétorique du discours dominant: « le discours normatif actuel qui postule la démocratie libérale et l'économie de marché comme ordre naturel du monde et stigmatise les utopies du $\mathrm{XX}^{\mathrm{E}}$ siècle ne laisse aucune place à la mélancolie de gauche $»^{22}$. À la lumière de ces considérations, nous ne souscrivons pas à l'opinion d'Yves Citton, qui invoque l'élaboration de nouvelles intrigues capables de séduire un électorat de gauche ${ }^{23}$; si un retour des narrations "de gauche» est souhaitable, cela ne doit pas passer par la recherche à tout prix d'une catharsis conciliatrice mais plutôt par la conception de narrations visant à inspirer une mélancolie naturelle chez les récepteurs. La production de formes d'écriture fragmentaires, hybrides, expérimentales, inachevées parce qu'inachevables, peut représenter une des formes de contestation les plus efficaces contre l'hégémonie actuelle du récit cathartique.

17 En conclusion, bien que la présence de narrations forgées à l'instar du modèle aristotélicien de la Poétique ne représente nullement un mal en soi, celle-ci pourrait produire des effets négatifs sur la vie d'une collectivité en empêchant l'existence de formes d'écriture alternatives. S'il paraît exagéré d'affirmer que toute mise en intrigue se greffe sur une idéologie d'extrême-droite, le contraire semble en revanche plausible, à savoir que toute narration d'extrême-droite se fonde sur une mise en intrigue déterminée au préalable. Les politiciens "de gauche», au lieu de fonder leur communication sur un programme de "fidélisation » des électeurs autour de la figure d'un leader aux traits messianiques, devraient plutôt approfondir la lecture des récits 
non-aristotéliciens, dont la charge subversive très puissante pourrait s'avérer une arme efficace à utiliser dans leur bataille politique :

D'abord refoulée par la gauche elle-même, puis stigmatisée à notre âge de restauration "postidéologique", cette mélancolie rebelle reste à découvrir, demande à être reconnue. Or mélancolie et révolution vont de pair. Comme une ombre, la mélancolie suit les pas de la révolution, se faisant discrète pendant son essor, ressurgissant après son épuisement et l'enveloppant après sa défaite ${ }^{24}$.

\section{NOTES}

1. C. Salmon, Storytelling, la machine à fabriquer des histoires et à formater les esprits, Paris, La Découverte, 2007.

2. «Tout se passe, me semble-t-il, comme si, dans nos démocraties privées de grands cadres herméneutiques et spirituels collectifs, le récit littéraire promettait de penser le singulier, de donner sens aux identités pluralisées, de retisser les géographies en constituant des communautés ", A. Gefen, Réparer le monde - La littérature française face au XXIe siècle, Paris, Édition Corti, 2017, p. 9.

3. E. Traverso, Mélancolie de gauche. La force d'une tradition cachée (XIX - XXIe siècle), Paris, Éditions La Découverte, 2016.

4. P. Ricœur, Temps et Récit - Tome I : l'intrigue et le récit historique, Paris, Seuil, 1983, p. 141.

5. Ibid. p. 80.

6. R. Baroni, La Tension narrative, suspense, curiosité et surprise, Paris, Seuil, 2007, p. 122.

7. V. Propp, Morphologie du conte, Paris, Seuil, 1970, p. 30.

8. J. Villeneuve, Le Sens de l'intrigue ou la narrativité, le jeu et l'invention du diable, Laval, Les Presses de l'Université Laval, 2004, p. 55.

9. R. Dupont-Roc et J. Lallot, notes à Aristote, Poétique, R. Dupont-Roc et J. Lallot (dir.), Paris, Édition du Seuil, 2011, p. 190.

10. H. R. Jauss, Pour une esthétique de la réception, Paris, Gallimard, 1978, p. 163.

11. M. Le Pen, « Discours de Bordeaux », 22 janvier 2012, cité dans C. Alduy, S. Wahnich, Marine le Pen prise aux mots. Décryptage du nouveau discours frontiste, Paris, Seuil, 2015, p. 125.

12. P.-A. Targuieff, "Nationalisme et réactions fondamentalistes en France. Mythologies identitaires et ressentiment antimoderne ", Vingtième Siècle. Revue d'histoire, no 25, 1990, pp. 49-74, pp. $64-65$.

13. "I consiglieri eletti del Movimento 5 Stelle come opposizione voteranno in Aula le proposte di buon senso indipendentemente da chi le presenta : le buone idee non sono né di destra nè di sinistra " (notre traduction). Paru sur Ilblogdellestelle.it, le 09 juin 2016, disponible ici: https:// www.ilblogdellestelle.it/2016/06/

il_movimento_5_stelle_non_da_indicazioni_di_voto_i_voti_sono_dei_cittadini.html (consulté le 09 décembre 2019).

14. «Presto ci saranno le elezioni le regionali [...] e vi dovrete confrontare con il voto degli italiani che vi hanno bocciato in tutte le elezioni degli ultimi anni [...] Non si può scappare per sempre. E quando ridarete la parola ai cittadini verrete spazzati via per sempre» (notre traduction). Discours prononcé au Sénat le 10 septembre 2019, disponible ici: https://corrieredibologna.corriere.it/bologna/politica/ 19_settembre_10/bibbiano-caos-aula-borgonzoni-provoca-attacco-pd-5a2bcafo-d3dc-11e9af08-7712fed3ba2e.shtml (consulté le 09 décembre 2019). 
15. C. Alduy, S. Wahnich, op. cit., pp. 154-155.

16. Jan-Werner Muller, Qu'est-ce que le populisme ?, Paris, Seuil, 2016, pp. 68-69.

17. A. Goudmand, « Narratologie du récit sériel », dans Proteus, $n^{\circ}$ 6, 2013, p. 81.

18. «Aujourd'hui, un réseau comme Netflix décide du genre de série qu'il produira sur la base de statistiques liées aux utilisateurs de son site », M. Marti, R. Baroni, « De l'interactivité du récit au récit interactif ", Cahiers de Narratologie, $\mathrm{n}^{\circ}$ 27, 2014.

19. N. Klein, No logo, Arles, Actes Sud, 2002, p. 53.

20. Entretien avec Sandra Laugier, paru sur Liberation.fr, le 18 octobre 2019, disponible ici : https://www.liberation.fr/debats/2019/10/18/sandra-laugier-les-series-sont-des-outils-deducation-de-pensee-et-de-combat-politique_1758416 (consulté le 22 avril 2020).

21. J. Kristeva, Soleil noir, dépression et mélancolie, Paris, Seuil, 1987, p. 264.

22. E. Traverso, op. cit., p. 223.

23. "Il faut que "la gauche" se remette à (se) raconter des histoires inspirantes, et il faut qu'elle arrache ou qu'elle crée les moyens de les faire circuler aussi largement que possible », Y. Citton, Mythocratie : storytelling et imaginaire de gauche, Paris, Éditions Amsterdam, 2010, p. 171.

24. E. Traverso, op. cit., p. 224.

\section{AUTEUR}

\section{ANTONINO SORCI}

Université Paris 3 - Sorbonne Nouvelle 